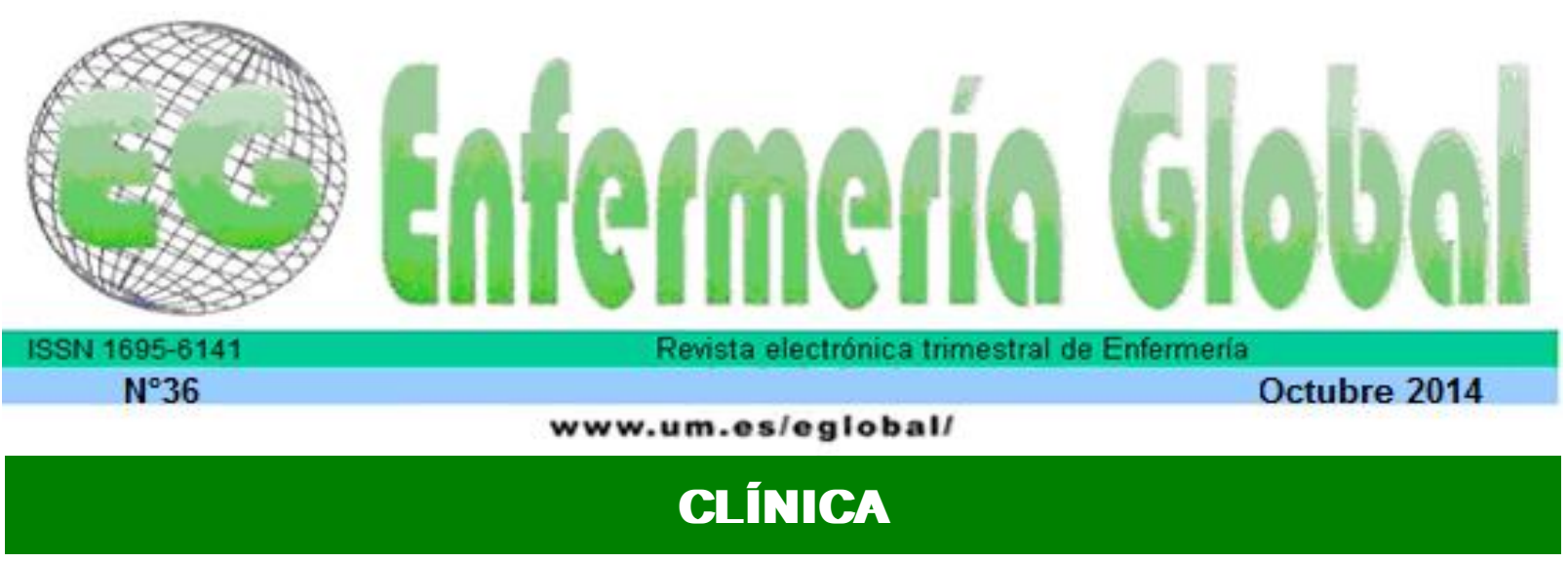

\title{
Promoción y prevención de la salud junto a los servicios de embellecimiento de manos y pies: inserción del enfermero
}

Promoção e prevenção da saúde junto aos serviços de embelezamento de mãos de pés: inserção do enfermeiro

Promotion and prevention of the health beside the services of beauty and aesthetics centers: insertion of the nurse

\section{*Peraça Vieira, Flávio **Heckler de Siqueira, Hedi Crecencia *** Sosa Silva José Richard de ****Cecagno, Diana}

*Enfermero Especialista. Maestria en Enfermería por la Universidad Federal de Rio Grande (FURG). Email: enfovieira@gmail.com **Doctora en Enfermería. Profesora del Programa de postgrado en Enfermería de maestría o doctorado (FURG) *** Doctorando en Enfermería del Programa de postgrado en Enfermería de maestría o doctorado (FURG), Profesor Facultad Anhanguera de Pelotas ****Doctoranda en Enfermería del Programa de postgrado en Enfermería de maestría o doctorado (FURG), Profesora Universidad Federal de Pelotas (UFPEL). Brasil.

Palabras clave: Promoción de la Salud; Centros de Belleza y Estética; Rol de la Enfermera; Equipos de Seguridad.

Palavras chave: Promoção da Saúde; Centros de Embelezamento e Estética; Papel do profissional de enfermagem; equipamentos de proteção.

Keywords: Health Promotion; Beauty and Aesthetics Centers; Nurse's Role and Protective Devices

\section{RESUMEN}

Objetivo: Evaluar el conocimiento de los profesionales del Servicio de Embellecimiento de Manos y Pies sobre la forma de transmisión de las Hepatitis B y C y verificar el uso de equipos de protección individual.

Metodología: Para obtener las informaciones se utilizó la investigación descriptiva-exploratoria con abordaje cualitativo. El estudio fue realizado en siete "salones de belleza" escogidos aleatoriamente por el método de sorteo. Los sujetos, identificados por seudónimos, fueron profesionales que estaban actuando en estos servicios, siendo escogido el más antiguo de cada "salón de belleza". La recolección de datos se realizó después de la aprobación por el Comité de Ética, bajo el número 186/2010, por el método de la entrevista semi-estructurada. 
Resultados: los datos demostraron que la mayoría no utiliza equipos de protección individual y expone al usuario a contaminación de patologías por desconocimiento de la vía de transmisión. Se concluye ser de suma importancia insertar en los cursos que preparan esos profesionales temas, abordados por profesionales de la salud, especialmente por el enfermero, sobre la promoción y prevención de esas patologías.

\section{RESUMO}

Objetiva-se avaliar o conhecimento dos profissionais do Serviço de Embelezamento de Mãos e Pés sobre a forma de transmissão das Hepatites $B$ e $C$ e verificar o uso de equipamento de proteção individual.

Metodologia; Para obter as informações utilizou-se o método descritivo-exploratório com abordagem qualitativa. O estudo foi realizado em oito "salões de beleza" escolhidos, aleatoriamente, pelo método de sorteio. Os sujeitos, identificados por pseudônimos, foram profissionais atuantes sendo escolhido o mais antigo de cada "salão de beleza". A coleta de dados aconteceu, após aprovação do Comitê de ética ํo 186/2010, pelo método da entrevista semi-estruturado.

Resultados: Os dados demonstraram que a maioria não utiliza equipamentos de proteção individual e expõe o usuário a transmissão de patologias por desconhecimento da via de transmissão. Infere-se ser de suma importância inserir nos cursos preparatórios desses profissionais temas, abordados por profissionais da saúde, especialmente pelo enfermeiro, sobre a promoção e prevenção dessas patologias.

\section{ABSTRACT}

Objective is to evaluate the knowledge of professional service Grooming Hands and Feet on the mode of transmission of Hepatitis $B$ and $C$ and check the use of personal protective equipment. To get the information we used the method descriptive exploratory qualitative approach.

Methodology: The study was conducted in seven "salons" chosen randomly by drawing lots. The subject, identified by pseudonyms, were active professionals being selected the oldest of seven "salon". The data collection was processed, after approval of the Ethics Committee No 186/2010, by the method of semi-structured interview.

Results: The data showed that the majority does not use personal protective equipment and expose the user to the transmission of diseases through ignorance of the transmission channel. It is inferred to be of paramount importance in the preparatory courses to enter these professional topics addressed by health professionals, especially by the nurse on the promotion and prevention of these diseases

\section{INTRODUCCIÓN}

La promoción de salud conforme la Organización Mundial de la Salud (OMS), comprende la población como un todo en el contexto de su día a día, en vez de enfocar grupos de riesgo para enfermedades específicas. Puede ser vista como una estrategia que se utiliza en la educación/aprendizaje para la producción de salud en el colectivo para responder a las necesidades de salud de la población.

Educar, del latín educare, significa conducir de un estado a otro, modificar en una determinada dirección, lo que es susceptible de aprendizaje. La educación comprende el conjunto de procesos, influencias, estructuras y acciones que intervienen en el desarrollo humano de individuos y grupos en su relación activa con el medio natural y social, en un determinado contexto de relaciones entre grupos y clases sociales, procurando la formación del ser humano. ${ }^{1}$ 
Como proceso, la educación no es una simple transmisión del conocimiento heredada de los antepasados para las nuevas generaciones, sino una tecnología capaz de producir una ruptura entre el saber sobrepasado e introducir el nuevo, más apropiado en la formación del ser humano, observando sus necesidades sociales y el medio en el cual vive, trabaja y se desarrolla.

El conocimiento permanece como una aventura en donde la educación debe suministrar el apoyo indispensable y así contribuir constantemente a mantener al ser humano actualizado y coherente en el tiempo y espacio que ocupa. ${ }^{2}$ Por lo tanto, la educación vista como un aprendizaje continuo, expresa una vinculación en la formación del sujeto, pues las exigencias del mundo digital y globalizado, provocan cambios constantes en la forma de pensar y actuar de las sociedades, introduciendo nuevos paradigmas y obligando su incorporación a los que desean sobrevivir y alcanzar éxito en sus actividades profesionales y personales. ${ }^{3}$ El proceso educacional puede ocurrir en el espacio no formal, o sea, aquel que sucede en ambientes y situaciones interactivas en las actividades cotidianas del ser humano, construyendo el conocimiento con la participación de los individuos. ${ }^{4}$

Se entiende que en el área de la salud los ambientes que merecen mayor atención educativa son los que atienden al usuario en situaciones de posibles vulnerabilidades como acontece en los Servicios de Embellecimiento de Manos y Pies (SEMP), porque existe la posibilidad de contaminaciones que pueden alcanzar al cliente por el uso de material compartido, por fallas en la desinfección/esterilización, por desconocimiento de las vías de transmisión y por descuido del propio profesional en la utilización de los equipos de protección individual (EPI).

Según la Norma Reguladora (NRR 4): se considera EPI todo dispositivo de uso individual destinado a preservar y proteger la integridad física del trabajador. ${ }^{5}$

En el SEMP existe probabilidad, de contaminaciones con el virus de hepatitis de los tipos B y C, capaces de agravios a la salud del individuo. La OMS estima que cerca de 2 billones de personas en el mundo ya tuvieron contacto con el virus de la hepatitis B y que 325 millones se hicieron portadores. ${ }^{6}$

El enfermero es un profesional que posee amparo legal, expresado en la Ley ㄲo 7 . 498, de 25 de junio de 1986 del ejercicio profesional, reglamentada por el Decreto no 94.406, de 08 de junio de 1987 para actuar en diversas áreas que envuelven la educación. Le cabe, igualmente, participar en la prevención y control de las enfermedades transmisibles en general y en los programas de vigilancia epidemiológica. Participa también, en programas y actividades de educación sanitaria, procurando la mejora de salud del individuo, de la familia y de la población en general. ${ }^{7}$

Por lo tanto, el enfermero en su actividad profesional ejerce entre otras funciones la actividad de educador de la salud ante la comunidad, con el objetivo, principalmente de trabajar la promoción y prevención en salud. La función del enfermero es estar con el otro, participando, aprendiendo, enseñando, investigando, articulando y, así, contribuyendo de forma integral en la promoción, prevención y también en la recuperación de la salud de los usuarios. Al actuar como articulador del cuidado y al interaccionar de forma activa y participativa en el pensar y hacer enfermería, colabora en la praxis de otros profesionales del equipo multiprofesional, teniendo como objetivo ser resolutivo y el bienestar de los usuarios de los servicios de salud. ${ }^{8}$ 
Se hace necesario entender la relación de la enseñanza e investigación, que muestra el camino a ser seguido para conocer el desconocido y posibilitar su descubrimiento:

"No hay enseñanza sin investigación e investigación sin enseñanza. Esos querer-hacer se encuentran uno en el cuerpo del otro. Mientras enseño continúo buscando, reprocurando. Enseño porque busco, porque indagué, porque indago y me indago. Investigo para constatar, constatando, intervengo, interviniendo educo y me educo. Investigo para conocer lo que aún no conozco y comunicar o anunciar la novedad." 9:32.

Al indicar que es preciso conocer el trabajo que es realizado por la sociedad para conocer lo que aún no se conoce, y a partir de ahí, comunicar o anunciar la novedad, induce al educador enfermero a reflexionar sobre las actividades cotidianas del trabajador en relación a los aspectos de su propia salud y también a los que prestan su servicio ${ }^{9}$. Es necesario verificar cómo él realiza su trabajo y constatar la necesidad o no de modificaciones en las actitudes y comportamientos para mantenerse saludable, a sí mismo y a los usuarios que presta servicio.

En el área de la salud, el equipo multiprofesional (EM) necesita proporcionar servicios que aseveren la integridad del ser humano, necesita observar las normas de vigilancia sanitaria y garantizar la seguridad al usuario/cliente que busca el servicio. En un sentido amplio el equipo debe buscar conocer los campos de actuación de los profesionales, cómo los productos y equipamientos son manipulados, y de qué forma estos servicios están siendo ofrecidos y realizados a la población.

Se percibe el EM como:

"una agrupación de individuos, con historias profesionales diferentes, lo que resulta en una heterogeneidad de conocimientos, con características y saberes propios, lo que exige una mirada y una interacción basada en la comprensión de la individualidad de cada componente."

En este sentido, la prevención de enfermedades y la promoción de la salud son constituidas como deber de todos los órganos de salud pública, fabricantes, empresarios y prestadores de servicios de embellecimiento, disponiendo y observando las normas de vigilancia sanitaria y de prácticas correctas en lo que se refiere a las instalaciones físicas, control de productos, medidas de higiene, limpieza y esterilización de materiales. ${ }^{10}$

Es preciso entender que la producción de la salud no se procesa solamente en la cura de la enfermedad, pero especialmente por medio de la prevención y promoción de la salud. Así siendo, la promoción de la salud en el local de trabajo, envuelve un conjunto de medidas que deben ofrecer oportunidades por el contratante, asumidas por el trabajador, custodiadas por la vigilancia sanitaria, e incentivadas por la sociedad, EM de salud, brindando más salud a todos.

Según el Consejo Federal de Enfermería compete al enfermero actuar en la prevención, recuperación y rehabilitación de la salud, con autonomía y en consonancia con los preceptos éticos y legales. En el artículo 70, responsabilidades y deberes, se destaca: "estimular, facilitar y promover el desarrollo de las actividades de 
enseñanza, investigación y extensión, debidamente aprobados por la institución."11 Por lo tanto, el enfermero es un profesional capacitado para trabajar medidas educativas, tanto en la comunidad, como en las Unidades Básicas de Salud (UBS), hospitales y también en proyectos realizados en escuelas o cursos de capacitación profesional, tales como de los SEMP y así, informar, esclarecer, enseñar y educar en salud para que en el futuro se tenga individuos más saludables.

Con base en ese contexto se buscó evaluar el conocimiento que el profesional del SEMP posee en relación al uso de EPI y la forma de transmisión de las Hepatitis B y $\mathrm{C}$ por posibles lesiones producidas durante la actividad profesional.

\section{METODOLOGÍA}

La investigación posee carácter descriptivo-exploratorio con abordaje cualitativo. La recolección de datos ocurrió después de aprobación del proyecto por el Comité de Ética de la Facultad Anhanguera bajo el número 186/2010. Inicialmente, se solicitó al ayuntamiento del municipio la lista de los "salones de belleza" que poseen SEMP. Después de adquirir esa relación, fue realizado el sorteo aleatorio de los siete "salones de belleza", para posteriormente entrar en contacto con los profesionales. Para eso fueron observados algunos criterios de inclusión: el salón de belleza debería tener profesional trabajando en el SEMP; poseer autorización de funcionamiento junto al ayuntamiento municipal; poseer curso y certificación profesional para este tipo de servicio y aceptar participar de la investigación permitiendo la divulgación de los datos.

Los datos fueron recolectados por medio de entrevista semi-estructurada, utilizando un cuestionario específicamente construido para esa finalidad. Los entrevistados tuvieron su identidad protegida, por seudónimos, optando por colores de esmaltes: Glamour Pink; Salon; Rosa Chique; Verde; Mix-Use; Azaléia y Rosa Amarilla. Con la intención de asegurar la fidelidad de los datos de las entrevistas estas fueron transcritas en su totalidad manteniéndose la secuencia en que acontecieron.

\section{PRESENTACIÓN Y DISCUSIÓN DE LOS RESULTADOS}

Para facilitar el análisis y discusión de los datos, fueron agrupados en dos categorías preestablecidas: uso de equipos de protección individual; formas de transmisión de la hepatitis $\mathrm{B}$ y $\mathrm{C}$.

\section{Uso de equipamiento de protección individual}

Para desarrollar la actividad profesional de embellecimiento de manos y pies está indicado usar guantes de procedimiento, lentes de protección y máscara. Los guantes de procedimiento deben ser usados tanto para el contacto con el usuario/cliente, así como en el momento de lavar el material y en la preparación para la esterilización. Las lentes de protección deben ser usadas en el momento de lavar los materiales, evitando el contacto de las soluciones con la mucosa ocular y también cuando haya riesgo de contaminación por sangre, fluidos y secreciones.

Al ser preguntadas si conocían o si fueron informadas sobre el uso de EPI (guantes de procedimiento, máscara y lentes de protección) para el proceso de lavado y desinfección de los materiales y si los utilizan: 06 entrevistadas respondieron que 
poseen informaciones, sin embargo, no hacen uso de los mismos, solamente 01 sujeto, Salon, confirmó hacer el uso solamente de guantes y máscara.

"Yo tuve informaciones por intermedio de revistas que leo, pero no las utilizo en el momento de lavar, utilizo más cuando estoy trabajando en un pie, en una mano, allí yo utilizo, pero en el momento de lavar me saco todo" (Glamour Pink).

"Sí, tengo conocimiento. Por los cursos que yo ya hice, e, incluso por un curso de noción en podología... Yo uso guante y máscara” (Salon).

"Sí, yo conozco porque la profesora explicó que es bueno usar. Es porque puede pasar algún hongo para mí o para otra persona y por eso tiene que ser usado. Pero yo no uso" (Rosa Chique).

"Sí, fui informada por la profesora del curso [...] Para no coger ningún tipo de enfermedad [...] Bacterias, hongos cosas así, pero no uso" (Verde).

Los sujetos relataron tener noción, pero solamente 01 hace el uso de dos de los tres EPI recomendados para protección. Salon usa guantes y máscara, no utiliza lentes de protección individual.

Glamour Pink dijo estar informada por los diarios y las revistas de moda, mientras Salon tiene conocimiento por los cursos de capacitación. Los otros sujetos relataron haber recibido informaciones de sus profesores del curso de embellecimiento de manos y pies.

Los 03 EPI son de gran importancia en el proceso de lavado y desinfección. La desinfección comprende la primera etapa para el proceso de limpieza del material. Ese proceso se da por medio del cepillado del material con agua y jabón, se enjuaga con agua corriente y se secan los materiales, para posteriormente ser sometidos a la esterilización. El guante es una barrera protectora de la piel en relación a los patógenos que pueden estar en los instrumentos de trabajo y también para evitar el contacto directo con las soluciones de limpieza, que pueden provocar procesos alérgicos al profesional en cuestión. ${ }^{5}$

La máscara de cirugía descartable debe ser usada tanto en la atención para evitar contacto con gotas de saliva liberadas por el cliente al hablar o toser. Además de eso, su uso es recomendado durante el proceso de desinfección, pues en el momento del contacto del material con agua y los productos utilizados para la desinfección, pueden salpicar y alcanzar la boca del profesional. ${ }^{12}$

Las lentes de protección de uso individual son recomendadas para ser utilizadas siempre que haya riesgo de contacto con sangre, secreción y fluidos. En ese caso el riesgo también está en el acto de limpiar el material, así el profesional protege su mucosa ocular. ${ }^{5}$

Al preguntar a los 07 sujetos entrevistados si hacían uso de guantes de procedimiento para atender a su clientela, 05 respondieron no que utilizaban, 01 que sí y 1 a veces. El cuadro $\mathrm{n}^{0} 1$ señala las respuestas y respectivas justificaciones. 
Tabla no 1 Uso de Guantes de Procedimiento

\begin{tabular}{|l|c|l|}
\hline SUJEITO & USA LUVA DE & JUSTIFICATIVA \\
\hline Glamour Pink & SI & $\begin{array}{l}\text { No me gusta entrar en contacto con los pies de las otras personas, y, } \\
\text { para no coger ningún tipo de hongo }\end{array}$ \\
\hline Salon & NO & Pierdo el tato para trabajar, prefiero no usar. \\
\hline Rosa Chique & NO & Pierdo el tato. \\
\hline Verde & A VECES & Uso sólo cuando estoy con mi uña pintada para manipular con los \\
& esmaltes. \\
\hline Mix-Use & NO & Pierdo el tato y me siento mal \\
\hline Azaléia & NO & Pierdo el tato. \\
\hline Rosa Amarela & Cuando hice mi curso aprendí a hacer sin guante, después queda ruin \\
& & de hacer con el guante porque parece que no tiene contacto. \\
\hline
\end{tabular}

Fuente: datos de la investigación organizados por Vieira, 2013.

Los guantes de procedimiento deben ser usados todas las veces que haya riesgo de contacto con sangre o secreciones. ${ }^{5}$ En este caso, el riesgo es inminente en cada procedimiento con los usuarios, porque nunca se sabe el momento en que podrá haber una lesión durante el trabajo. En ese sentido, su uso debe ser constante y en todos los procedimientos junto al usuario y también al realizar la limpieza de los materiales utilizados, que pueden estar contaminados con hongos, bacterias patógenas.

Destaca el gran número de sujetos que no hacen uso del guante al atender al usuario/cliente. De las 07 participantes de la investigación 05 entrevistadas respondieron negativamente al uso de guantes durante el procedimiento, justificando que pierden el tacto. Se percibe la gravedad de no usarlo Más preocupante fue la respuesta de Rosa Amarilla, que demostró falta de conocimiento, por no haber recibido las informaciones necesarias en la institución en la cual realizó el curso de embellecimiento de manos y pies, pues afirmó que "aprendió en el curso a hacer sin el guante".

El guante supone una barrera de protección en la piel del profesional, si existe alguna lesión en el transcurso de la atención al cliente liberando sangre o secreción. Evita el contacto directo y consecuentemente la transmisión de agentes patógenos. Es deber del profesor de cursos en esta área de estética, explicar y enfatizar a sus alumnos las formas de contagio de las enfermedades y mostrar que el simple uso de un par de guantes protege su salud. Los futuros profesionales sólo tendrán el hábito del uso de EPI, al iniciar a utilizarlos en las clases prácticas, para que durante los primeros contactos con los usuarios ya lo hagan con destreza y total seguridad, adecuándose y desarrollando habilidades para realizar la práctica de su trabajo. Solamente de esta manera lo llevarán a su cotidiano. Conviene resaltar que, entre uno y otro cliente, es preciso realizar el cambio de guantes y la higienización de las manos con agua y jabón. 
La máscara debe ser usada siempre que se atienda un cliente, debido a la proximidad entre ambos. Durante el tratamiento estético el cliente libera gotas de saliva al hablar, toser o estornudar. Estando el cliente contaminado por un patógeno podrá transmitirlo al profesional del SEMP. Entre las enfermedades transmitidas por gotitas se destacan la Tuberculosis; Papera; Infección por Influenza A; Meningitis por Haemophylus influenzae, Rubéola entre otros. ${ }^{12}$

\section{Formas de transmisión de las hepatitis B y C}

En relación a las formas de transmisión de las Hepatitis $\mathrm{B}$ y $\mathrm{C}$, la gran mayoría, 06 afirmaron conocerlas, sólo una de las entrevistadas afirmó no saber. De los siete participantes entrevistados solamente Verde no conocía ninguna de las formas de transmisión y Mix-use no conocía la transmisión de la Hepatitis C. Los otros sujetos presentaron algún conocimiento en relación a esa cuestión (Tabla $n^{\circ} 2$ ). Se percibe que en relación a la Hepatitis B 02 sujetos afirman que el contacto se da directo por relación sexual, 02 dicen que se procesa por el contacto directo con la sangre, 01 por el contacto directo con la sangre y por relaciones sexuales, 01 sujeto dice que la transmisión ocurre por relación sexual, contacto directo con la sangre y saliva y solamente 01 no supo informar.

Tabla 2: Transmisión Hepatitis B y C.

\begin{tabular}{|c|c|l|}
\hline SUJETO & HEPATITIS B & \multicolumn{1}{c|}{ HEPATITIS C } \\
\hline $\begin{array}{c}\text { Glamour } \\
\text { Pink }\end{array}$ & $\begin{array}{c}\text { Sexualmente, contacto directo con sangre y } \\
\text { saliva. }\end{array}$ & $\begin{array}{l}\text { Todavia no sé [...] Pienso que da la misma } \\
\text { forma }\end{array}$ \\
\hline Salon & Sexualmente & Sangre [...] Por un corte. \\
\hline $\begin{array}{c}\text { Rosa } \\
\text { Chique }\end{array}$ & Sangre & Sangre \\
\hline Verde & No & No \\
\hline Mix - Use & Sexual & No se \\
\hline Azaléia & Contacto directo con sangre. & Contacto directo con sangre. \\
\hline $\begin{array}{c}\text { Rosa } \\
\text { Amarela }\end{array}$ & Contacto directo con sangre y relaciones \\
sexuales & Contacto con sangre \\
\hline
\end{tabular}

Fuente: datos de la investigación organizados por Vieira, 2013.

Un levantamiento hecho por el Ministerio de la Salud entre 1999 y 2009 y divulgado en la primera conmemoración del Día Mundial del Combate a las Hepatitis Virales revela que, en ese periodo, fueron confirmados 96.044 casos de la enfermedad causados por el virus B. De ese total, más del $50 \%$ alcanzaron personas entre 20 y 39 años. El estudio muestra también que las confirmaciones de Hepatitis $C$ fueron 60.908. Este número elevado de casos confirmados de hepatitis de los tipos $\mathrm{B}$ y $\mathrm{C}$ en todo el país, entre 1999 y 2009, preocupa al Ministerio de Salud. El profesional de salud, en el que se incluyen los enfermeros, deben, según compromiso social profesional asumido, empeñarse en disminuir esos índices y velar por la promoción y prevención de la salud de la población. ${ }^{3}$

El proceso de educación como un proceso continuo de adquisición de saberes y habilidades, cualificaciones y aptitudes posibilita al individuo, a través de la reflexión crítica de la realidad, a mejorar su condición de existir en el mundo, así como a cooperar e influir, positivamente, a los que forman parte de su cotidiano 


\section{CONCLUSIÓN}

Durante la recolección de datos y, posteriormente en su análisis e interpretación, se constató fragilidades en las respuestas de los sujetos en relación al uso de los EPI y las formas de transmisión de las hepatitis. Esa constatación conduce a considerar la falta de protección y seguridad de la salud del usuario y también del propio trabajador de los SEMP. Se hace necesario que ocurra una evaluación de los cursos, así como de los currículos desarrollados en los mismos que preparan esos profesionales. Debe ser revisada, también, la forma de abordaje de los conocimientos relativos al uso de protección en la atención al cliente y en el proceso de desinfección de los materiales, y así, ofrecer seguridad a la salud de los trabajadores y usuarios de esos servicios.

Los SEMP son servicios que atienden diversos individuos, que pueden o no estar con alguna patología infectocontagiosa. En los casos positivos existe la probabilidad de transmisión por material contaminado a los usuarios que frecuentan esos servicios. En este sentido, se destaca la necesidad de la actuación de profesionales de salud para trabajar los temas relacionados con las técnicas de promoción de la salud y prevención de agravios. Los usuarios de estos servicios, infectados con algún agente patógeno, al compartir el material son capaces de diseminarlos para incontables personas, causando problemas a la salud, sufrimiento y repercusión negativa en el sistema de salud, elevación de valores y daños en la calidad de vida del usuario.

Se alerta a los gobiernos locales que, junto a las secretarías de salud, vigilancia sanitaria y educación pueden y deben crear estrategias de capacitación para estos profesionales que se encuentran en diversos lugares frecuentados por miles de usuarios de forma constante y se encuentran, conforme datos de esa investigación, vulnerables a la contaminación de agentes patógenos por falta de uso adecuado del material utilizado y descuido de las normas de seguridad.

El EM de salud, integrado por diversos profesionales, entre ellos el enfermero, posee habilitación legal para participar de forma activa y permanente promoviendo encuentros, palestras y así divulgando el conocimiento y buscando proteger a los profesionales y a los usuarios/clientes de estos servicios. Además, es preciso incluir en los cursos de preparación de profesionales de los SEMP conocimientos teóricos y prácticos sobre desinfección/esterilización de material utilizado, uso de EPI y estrategias de promoción de la salud y prevención de enfermedades.

Se recomienda la inclusión del enfermero en los cursos de preparación de esos profesionales para desarrollar temas relativos a la promoción de la salud y prevención de patologías. A los profesionales que ya actúan se sugiere cursos de actualización con temas que permiten sensibilizarlos y concienciarlos de la necesidad de proteger tanto a los usuarios como a sí mismos, para una vida más saludable y de mayor calidad por medio de la educación.

Se enfatiza que la educación es un proceso que propicia condiciones al individuo para adquisición de conocimientos, para que él alcance su desarrollo personal y profesional. En este sentido, se destaca como indispensable estructurar y desarrollar junto a los profesionales o candidatos que se preparan para ejercer la profesión, cursos sobre temas de microbiología, formas de transmisión de enfermedades infectocontagiosas, necesidad de protección individual y el manejo correcto de la desinfección de los materiales utilizados en el embellecimiento de manos y pies y principalmente estrategias de promoción de la vida. 
El conocimiento construido con esta investigación podrá ayudar otros trabajos y también servir de alerta a los profesionales Enfermeros, mostrando la necesidad de ocupar espacios que se encuentran vacíos, pero posibles de ser ocupados por estos. Esta es una de las maneras con las que puede y debe colaborar con la salud de la población, expuesta a incontables problemas, como cuando busca el servicio de embellecimiento de manos y pies.

En relación al tema, se reitera la necesidad de nuevas investigaciones con la finalidad de profundizar en los conocimientos, y de esta forma, avanzar en esa temática que posee gran significancia en el campo de la salud pública y, ciertamente, diversos aspectos aún no fueron detectados, ni debidamente esclarecidos.

\section{REFERENCIAS}

1.LIBÂNEO, J. C. Pedagogia e pedagogos: inquietações e buscas educar. Curitiba: UFPR, 2001.

2. MORIN, E. Os sete saberes necessários à educação do futuro. $2^{\underline{a}}$ ed. São Paulo: Cortez/UNESCO; 2011.

3. BRASIL, Ministério da Saúde. Política Nacional de Educação Permanente em Saúde, Série B. Textos Básicos de Saúde.Série Pactos pela Saúde 2006, v. 9. Brasília - DF 2009.

4. FARIA, R. L. de; JACOBUCCI, D. F. C.; OLIVEIRA, R.C. Possibilidades de ensino de botânica em um espaço não-formal de educação na percepção de professoras de ciências. Rev. Ensaio. V.13. nำ1. Belo Horizonte:2011.

5. Agência Nacional de Vigilância Sanitária (ANVISA). Norma regulamentadora 4. Disponível para acesso em: www.anvisa.gov.br. Acessado em: 21 fev. 2013.

6. FERREIRA, C.T.; SILVEIRA, T.R. Hepatites virais: aspectos da epidemiologia e da prevenção. Rev. Bras. Epidemiol. 2004;7(4):473-87.

7. BRASIL. Lei 7.498 de 25 de junho de 1986. Dispõe sobre a regulamentação do exercício da Enfermagem e dá outras providências. Disponível para visualização em: http://portal.saude.gov.br/portal/saude/visualizar texto.cfm?idtxt=26973. Acesso em 20 de dezembro de 2012.

8. SIQUEIRA, H. C. H.; CECAGNO, D.; PEREIRA, Q. L. C (org). Equipe multiprofissional de saúde: ações inter-relacionadas. Pelotas/RS: Editora e Gráfica Universitária, 2009.

9. FREIRE, P. Pedagogia da Autonomia: saberes necessários a prática edcuativa. 43a ed. São Paulo. Paz e Terra, 2011.

10. FIORENTINI, S.R.B. Exigência da Vigilância Sanitária para Salão de Beleza: Beleza com Segurança. Disponível em: http://www.hairbrasil.com/congresso/sebrae2009/fiorentini.pdf. Acesso em 20 abr. 2013.

11. Código de Ética dos Profissionais de Enfermagem. Goiânia/GO: AB Editora, 2012. 12. GREGORCIC, A; at al. Cartilha de Proteção Respiratória contra Agentes Biológicos para Trabalhadores de Saúde. Disponível em: http://www.anvisa.gov.br/divulga/public/cartilha mascara.pdf . Acesso em 22 fev. 2013.

ISSN 1695-6141

๑) COPYRIGHT Servicio de Publicaciones - Universidad de Murcia 